Published in final edited form as:

Anal Chem. 2017 April 18; 89(8): 4428-4434. doi:10.1021/acs.analchem.6b03675.

\title{
Ubiquitin Chain Enrichment Middle-Down Mass Spectrometry Enables Characterization of Branched Ubiquitin Chains in Cellulo
}

\author{
Sean O. Crowe $\rrbracket^{\Uparrow, \ddagger, \dagger}$, Ambar S. J. B. Rana ${ }^{\Uparrow, \ddagger, \dagger}$, Kirandeep K. Deolף, Ying Ge ${ }^{\ddagger, \S, \|}$, and Eric R. \\ Strieter ${ }^{*}, \mathbb{\Upsilon}, \perp$ \\ IDepartment of Chemistry, University of Massachusetts-Amherst, Amherst, Massachusetts \\ 01003, United States \\ ‡Department of Chemistry, University of Wisconsin-Madison, Madison, Wisconsin 53706, United \\ States \\ §Department of Cell and Regenerative Biology, School of Medicine and Public Health, University \\ of Wisconsin-Madison, Madison, Wisconsin 53706, United States \\ "Human Proteomics Program, University of Wisconsin-Madison, Madison, Wisconsin 53705, \\ United States \\ ${ }^{\perp}$ Department of Biochemistry and Molecular Biology, University of Massachusetts-Amherst, \\ Amherst, Massachusetts 01003, United States
}

\begin{abstract}
Ubiquitin (Ub) has a broad functional range that has been ascribed to the formation of an array of polymeric ubiquitin chains. Understanding the precise roles of ubiquitin chains, however, is difficult due to their complex chain topologies. Branched ubiquitin chains are particularly challenging, as multiple modifications on a single ubiquitin preclude the use of standard bottomup proteomic approaches. Developing methods to overcome these challenges is crucial considering evidence suggesting branched chains regulate the stability of proteins. In this study, we employ Ubiquitin Chain Enrichment Middle-down Mass Spectrometry (UbiChEM-MS) to identify branched chains that cannot be detected using bottom-up proteomic methods. Specifically, we employ tandem ubiquitin binding entities (TUBEs) and the K29-selective Np14 Zinc Finger 1 (NZF1) domain from the deubiquitinase TRABID to enrich for chains from human cells. Minimal
\end{abstract}

\footnotetext{
*Corresponding Author: estrieter@umass.edu.

$\dagger$ Author Contributions

S.O.C. and A.S.J.B.R. contributed equally to this work.

Supporting Information

The Supporting Information is available free of charge on the ACS Publications website at DOI: 10.1021/acs.analchem.6b03675. Optimization of ubiquitin chain enrichments, additional MG132 treated experiments used to generate bar graphs in Figures 2 and 3, additional MS2 fragments supporting the assignment of K29 and K48 modifications, and supporting experimental methods (PDF)

ORCID

Ying Ge: 0000-0001-5211-6812

Eric R. Strieter: 0000-0003-3447-3669

Notes

The authors declare no competing financial interest.
} 
trypsinolysis followed by high resolution mass spectrometric analysis reveals that $\mathrm{Ub}$ chain branching can indeed be detected using both Ub binding domains (UBDs) tested at endogenous levels. We find that $\sim 1 \%$ of chains isolated with TUBEs contain Ub branch points, with this value rising to $\sim 4 \%$ after proteasome inhibition. Electron-transfer dissociation (ETD) analysis indicates the presence of K48 in these branched chains. The use of the NZF1 domain reveals that $\sim 4 \%$ of the isolated chains contain branch points with no apparent dependence on proteasome inhibition. Our results demonstrate an effective strategy for detecting and characterizing the dynamics of branched conjugates under different cellular conditions.

\section{Graphical abstract}

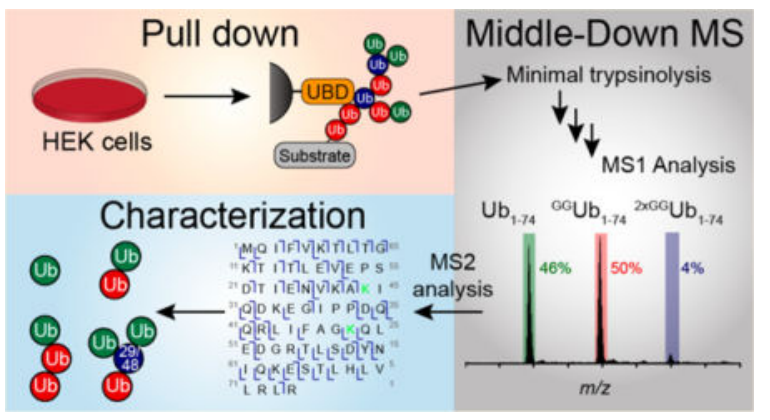

Over the last few decades, it has become widely appreciated that ubiquitin (Ub) plays a major role in regulating nearly every cellular pathway in eukaryotes. The cell cycle, for example, is driven by Ub-mediated degradation; ${ }^{1}$ the cellular response to DNA damage ${ }^{2}$ and our ability to ward off pathogens are also both intricately linked to Ub signaling. ${ }^{3}$ How Ub regulates the dynamics of such diverse biochemical pathways is thought to be a result of $\mathrm{Ub}$ chain formation.

Ub chains are generated after consecutive rounds of target protein modification by E1, E2, and $\mathrm{E} 3$ enzymes. ${ }^{4}$ After $\mathrm{Ub}$ is transferred to the $\varepsilon$-amino group of a target lysine, any of the eight amino groups of $\mathrm{Ub}(\mathrm{M} 1, \mathrm{~K} 6, \mathrm{~K} 11, \mathrm{~K} 27, \mathrm{~K} 29, \mathrm{~K} 33, \mathrm{~K} 48$, and K63) can be attached to the C-terminus of another $\mathrm{Ub}$ leading to the formation of a Ub chain. ${ }^{5}$ During chain extension, there can be tremendous variability in linkage, length, and configuration (i.e., linear versus branched). This versatility is what likely gives rise to the multitude of cellular functions associated with $\mathrm{Ub}$. Mounting evidence suggests that the type of ubiquitin chain determines the cellular fate of a modified protein. For instance, K11- and K48-linked chains signal for protein degradation through the proteasome, whereas M1- and K63-linked chains promote proteasome-independent activities. ${ }^{6-15}$

While there has been significant interest in understanding the functional impact of different chain linkages, much less attention has been paid to the importance of chain configuration. Initial reports on branched chains focused on bifurcations at consecutive lysines of Ub (e.g., K6/K11, K27/K29, or K29/K33) since these modifications can be detected using bottom-up proteomic approaches. ${ }^{16,17}$ On the basis of in vitro proteasome assays, bifurcated chains were found to be less effective at promoting protein degradation compared to chains composed of a single linkage. ${ }^{16,18}$ Whether these observations are solely due to chain 
branching, however, is unclear given the heterogeneity of the systems under investigations. More recently, there has been evidence suggesting K11/K48 branched chains produced by the anaphase-promoting complex/cyclosome (APC/C) are capable of triggering proteasomal degradation. ${ }^{19,20}$ In this case, K11/K48 branched chains were detected on APC/C substrates by expressing nonnative $\mathrm{Ub}$ variants in cells. Other branched chains unamenable to tryptic fragment mass spectrometric analyses have also been implicated in several fundamental cellular pathways. For example, M1/K63 chains have been proposed to regulate NF- $x$ B signaling; ${ }^{21,22} \mathrm{~K} 11 / \mathrm{K} 63$ conjugates are thought to play a role in receptor internalization, ${ }^{23,24}$ and K29/K48 branched chains have been linked to proteasomal degradation. ${ }^{25,26}$ Further defining the roles of branched chains in regulating protein stability and other cellular pathways requires new methods of detection and characterization.

In this study, we demonstrate the utility of Ubiquitin Chain Enrichment Middle-down Mass Spectrometry (UbiChEM-MS) in characterizing branched Ub chains present in human cell extracts. Previous reports from our lab and others have shown that, when integrated with MS analysis, minimal trypsinolysis and limited acid cleavage retain information on the connectivity of Ub chains, particularly branched chains. ${ }^{27-31}$ When Ub is subjected to trypsinolysis under nondenaturing conditions, a single cleavage occurs between Arg74 and Gly75 leaving behind an intact $\mathrm{Ub}_{1-74}$ fragment (calcd 8450.57 Da) as well as a Gly-Gly remnant. ${ }^{32}$ In the event of minimal trypsinolysis of Ub chains, this Gly-Gly remnant remains attached to the modified lysine resulting in a $\mathrm{Ub}_{1-74}$ fragment modified with a single GlyGly ( ${ }^{\mathrm{GG}} \mathrm{Ub}_{1-74}$, calcd $8564.62 \mathrm{Da}$ ). If a branch point is present within the Ub chain, the $\mathrm{Ub}$ moiety bearing the branch point will be modified with two Gly-Gly modifications $\left(2 \times \mathrm{XG} \mathrm{Ub}_{1-74}\right.$, calcd $\left.8678.66 \mathrm{Da}\right)$, thus enabling characterization of multiple modifications on a single Ub protein. Although useful for characterizing the products of E2-E3 enzymatic activity in vitro, these middle-down methods have not led to the identification of branched chains in complex mixtures such as cell extracts. We envisioned that, by lysing cells grown under different conditions and enriching particular $\mathrm{Ub}$ chains using linkage-selective $\mathrm{Ub}$ binding domains (UBDs), ${ }^{5,33}$ branched conjugates could be detected and characterized by middle-down MS. Here, we show this strategy is brought to fruition using both the nonselective tandem ubiquitin binding entities (TUBEs) and the K29 linkage-selective Npl4 Zinc Finger 1 (NZF1) domain from the deubiquitinase TRABID. Our results reveal that UbiChEM-MS can be used to not only detect previously undetectable branched chains but also monitor their dynamics. We surmise this same approach can readily be adapted for the detection and characterization of many other branched chains, provided a linkage specific binding domain exists.

\section{EXPERIMENTAL SECTION}

\section{Preparation of Halo-NZF1 Resin}

HaloLink resin ( $1.6 \mathrm{~mL}$, Promega) was pelleted at $800 \mathrm{~g}$ for $2 \mathrm{~min}$, and the supernatant was removed. The resin was washed four times with binding buffer $(2 \mathrm{~mL} ; 50 \mathrm{mM}$ Tris, $150 \mathrm{mM}$ $\mathrm{NaCl}, 10 \%$ glycerol, $0.05 \%$ IGEPAL CA-630) and then finally resuspended in binding buffer to yield a $50 \%$ slurry. This slurry was then incubated with $800 \mu \mathrm{L}$ of Halotag-NZF1 fusion protein $(115 \mu \mathrm{M})$, and the resulting suspension was rocked overnight at $4{ }^{\circ} \mathrm{C}$. The 
suspension was then pelleted at $800 \mathrm{~g}$ for $2 \mathrm{~min}$; the supernatant was discarded, and the resin was further washed four times with $5 \mathrm{~mL}$ of binding buffer.

\section{Isolation of Ubiquitin Chains from HEK Cell Lysate}

Resin bearing the appropriate ubiquitin binding domain (100 $\mu \mathrm{L}$ of TUBEs or $200 \mu \mathrm{L}$ of NZF1) was incubated with $45 \mathrm{mg}$ of cell lysate on a revolver overnight at $4{ }^{\circ} \mathrm{C}$, after which the resin was pelleted at $800 \mathrm{~g}$ for $2 \mathrm{~min}$. The resin was then washed three times with $2 \mathrm{~mL}$ of binding buffer and twice with $2 \mathrm{~mL}$ of minimal buffer $(50 \mathrm{mM}$ Tris, $150 \mathrm{mM} \mathrm{NaCl}, \mathrm{pH}$ 7.5). The resin was then resuspended in $100 \mu \mathrm{L}$ of minimal buffer. $6 \times$ Laemmli loading buffer was added to aliquots of each step, separated on a 15\% SDS-PAGE gel, and analyzed by Western blot with anti-Ub antibody (P4D1).

\section{On-Resin Minimal Trypsinolysis of Isolated Ub Chains}

Minimal trypsinolysis was performed on-resin at room temperature for $16 \mathrm{~h}$ using empirically determined ratios of lysate to trypsin (Table S1). These on-resin samples were then acidified to $\mathrm{pH} 2$ with acetic acid to deactivate trypsin. These acidified solutions were incubated at $4{ }^{\circ} \mathrm{C}$ for $15-30 \mathrm{~min}$ and then centrifuged for $5 \mathrm{~min}$ at $13000 \mathrm{~g}, 4^{\circ} \mathrm{C}$. For samples analyzed by LC MS/MS, the supernatant was concentrated $\sim 5-7$-fold using a speedvac. Samples were centrifuged for $30 \mathrm{~min}$ at $16000 \mathrm{~g}, 4^{\circ} \mathrm{C}$, prior to injection into the mass spectrometer. For samples analyzed by direct infusion into the mass spectrometer, the supernatant was pipetted into a $100 \mathrm{mg} \mathrm{SeP-Pak} \mathrm{C}_{18}$ column (Waters) which was washed with $10 \mathrm{~mL}$ of equilibration solution ( $0.1 \%$ TFA in water). This column was then washed with $3 \mathrm{~mL}$ of equilibration solution, followed by collecting $1.5 \mathrm{~mL}$ fractions of $10 \%, 20 \%$, $30 \%, 40 \%, 50 \%, 60 \%, 70 \%$, and $100 \%$ acetonitrile in $0.1 \%$ TFA solutions. The $0-60 \%$ fractions were lyophilized. Lyophilized samples from the $30 \%$ and $40 \%$ fractions were dissolved in $200 \mu \mathrm{L}$ of a water/acetonitrile/acetic acid (45:45:10) solution and analyzed by MALDI-TOF. Then, the samples containing Ub species were further analyzed by high resolution mass spectrometry.

\section{Middle-Down Mass Spectrometry and Quantitative Analysis with Orbitrap Fusion}

Samples were directly infused into an Orbitrap Fusion Tribrid mass spectrometer (Thermo Scientific Inc.), where the resolving power of the mass analyzer was set at 60000 . All spectra were processed with in-house software (MASH Suite ${ }^{34}$ ) using a signal-to-noise $(\mathrm{S} / \mathrm{N})$ threshold of 3 and a fit factor of $70 \%$ and then validated manually; all reported calculated and experimental values correspond to the most abundant molecular weights. Quantitative analysis of the relative abundance of the three $\mathrm{Ub}_{1-74}$ species was performed as described previously. ${ }^{35,36}$ The relative abundances of each $\mathrm{Ub}$ species in the $700-1300 \mathrm{~m} / \mathrm{Z}$ range were averaged to obtain the relative percentage. Percent distribution was calculated from three biological replicates, and data were represented as mean \pm standard error of the mean (SEM). 


\section{Liquid Chromatography Middle-Down Mass Spectrometry and Quantitative Analysis with Bruker Maxis Impact LCMS System}

Minimally digested products were separated using a nanoAcquity LC system (Waters) equipped with a replacement capillary custom packed trap (nanoLCMS Solutions LLC) followed by a home-packed PLRP-S column and a gradient from 5\% B to 95\% B over 43 min (solvent $\mathrm{A}, 0.1 \%$ formic acid in water; solvent $\mathrm{B}, 0.1 \%$ formic acid in $1: 1$ solution of acetonitrile and ethanol). The LC system was coupled online with a Bruker Impact II Q-TOF mass spectrometer, where the resolving power of the mass analyzer was set at 50000 . All spectra were processed with in-house software (MASH Suite ${ }^{34}$ ) using a signal-to-noise $(\mathrm{S} / \mathrm{N})$ threshold of 3 and a fit factor of $70 \%$ and then validated manually; all reported calculated and experimental values correspond to the most abundant molecular weights. Quantitative analysis of the relative abundance of the three $\mathrm{Ub}_{1-74}$ species was performed as described previously. ${ }^{35,36}$ The relative abundances of each Ub species in the $600-1250 \mathrm{~m} / \mathrm{Z}$ range were averaged to obtain the relative percentage. The percent distribution was calculated from three biological replicates, each with three technical replicates, and data were represented as the mean \pm standard error of the mean (SEM).

\section{Electron-Transfer Dissociation (ETD) Analysis of Ub Chain Linkages on Orbitrap Fusion}

For tandem mass spectrometry (MS/MS) experiments, individual charge states of protein molecular ions were isolated and then dissociated by ETD using a $10 \mathrm{~ms}$ reaction time, with a $2.0 \times 10^{5}$ reagent ion target. ETD was also supplemented by collision induced decay (CID) using a collision energy of $10 \%$. Spectra were recorded in the Orbitrap analyzer over an $\mathrm{m} / \mathrm{z}$ range of 150-2000 with a resolution setting of 60000 and AGC setting of $4 \times 10^{5}$ charges. All spectra were processed with the MASH suite software ${ }^{34}$ using a S/N threshold of 3 and a fit factor of $70 \%$ and then validated manually. The resulting mass lists were further assigned on the basis of the protein sequence of Ub with or without the diglycine (GG) modification at each lysine using a tolerance of $20 \mathrm{ppm}$ for precursor and fragment ions. All reported calculated (calcd) and experimental (exptl) values correspond to the monoisotopic molecular weight.

\section{ETD Analysis of Ub Chain Linkages on Bruker Maxis Impact LCMS System}

Minimally digested products were separated using a nanoAcquity LC system (Waters) equipped with replacement capillary custom packed trap (nanoLCMS Solutions LLC) followed by a home-packed PLRP-S column and a gradient from 5\% B to 95\% B over 43 min (solvent A: $0.1 \%$ formic acid in water; solvent B: $0.1 \%$ formic acid in $1: 1$ solution of acetonitrile and ethanol). The LC system was coupled online with a Bruker maXis II ETD Q-TOF mass spectrometer. For tandem mass spectrometry (MS/MS) experiments, individual charge states of protein molecular ions were isolated; then, the ions were dissociated by ETD. The accumulation time, reagent time, and reaction time for ETD were determined on a case-by-case basis to achieve optimal fragmentation. The ranges for accumulation time, reagent time, and reaction time were 1000-4000, 4-20, and 1-10 ms, respectively. All spectra were processed with the MASH suite software ${ }^{34}$ using a S/N threshold of 3 and a fit factor of $70 \%$ and then validated manually. The resulting mass lists were further assigned on the basis of the sequence of $\mathrm{Ub}$ with or without the diglycine (GG) modification at each 
lysine using a tolerance of $20 \mathrm{ppm}$ for precursor and fragment ions. All reported calculated (calcd) and experimental (exptl) values correspond to the monoisotopic molecular weight.

\section{RESULTS AND DISCUSSION}

A general characterization method is needed to understand the various roles that branched Ub chains play in cell signaling. To this end, we sought to develop an approach based on middle-down MS. ${ }^{27,28}$ We envisioned an experimental workflow commencing with the isolation of $\mathrm{Ub}$ chains from cells followed by minimal trypsinolysis and MS analysis (Figure 1). While there are several methods to enrich Ub chains using Ub binding domains (UBDs), we focused on two UBDs in particular: the nonselective tandem ubiquitin binding entity TUBE $2^{37}$ and the NZF1 domain from the K29/K33 selective deubiquitinase TRABID. ${ }^{38}$ We reasoned these two UBDs would allow us to monitor both global changes in Ub chain branching and changes in specific Ub chains bearing branched linkages.

\section{TUBE2- and NZF1-Based UbiChEM-MS}

To gain an understanding of the global landscape of Ub chain architecture, we captured polyubiquitin chains using the commercially available agarose bound TUBE2. This tandem ubiquitin binding entity is based on the UBA1 domain of $\operatorname{Rad} 23$ a and has been shown to enrich for Ub chains with little linkage selectivity, thus allowing us to monitor global changes in the Ub pool. ${ }^{37,39}$ Incubation of TUBEs with clarified lysate from HEK293 cells did indeed result in the isolation of high molecular weight chains (Figure S1). After washing, trypsin was added to achieve minimal digestion of the immobilized chains. The resulting digests were then subjected to reverse-phase column chromatography to remove salts and smaller peptides prior to high resolution MS analysis. With this strategy, we could detect all three $\mathrm{Ub}_{1-74}$ species in untreated HEK293 cell lysates (Figure 2A). Relative quantification reveals that $57 \%$ of $\mathrm{Ub}_{1-74}$ species exist as either caps or monoUb, $41 \%$ are ${ }^{\mathrm{GG}} \mathrm{Ub}_{1-74}$, and $2 \%$ are ${ }^{2 \times G G} \mathrm{Ub}_{1-74}$.

Having established a method that enables characterization of the Ub chain landscape, we wanted to explore whether UbiChEM-MS could be used to monitor chain dynamics. Branched Ub chains have been implicated as potent signals for proteasomal degradation, suggesting that proteasome inhibition would lead to an accumulation of these conjugates. ${ }^{20}$ To test this, cells were treated with MG132 for 2, 4, 8, and $16 \mathrm{~h}$ and chains were enriched using TUBE2 (Figures 2 and S3 and Table S2). The most significant changes occur after 16 h. Caps/monoUb decreases from $57 \%$ to $44 \%$, while $\mathrm{GG}^{\mathrm{Ub}} \mathrm{b}_{1-74}$ increases from $41 \%$ to $52 \%$ and ${ }^{2 \times G G} \mathrm{Ub}_{1-74}$ rises from $1 \%$ to $4 \%$ (Figure $2 \mathrm{~B}$ ). These results indicate that long chains harboring branch points accumulate after prolonged proteasome impairment, supporting the idea that branched chains are involved in proteasomal degradation.

We then shifted our attention to a UBD with linkage selectivity to capture a specific pool of Ub chains. The NZF1 domain from the deubiquitinase TRABID selectively binds K29linked $\mathrm{Ub}$ chains and has been shown to enrich heterotypic Ub chains from cell lysates. ${ }^{38}$ On the basis of these results, we reasoned that a percentage of mixed linkage chains would contain branch points and the dynamics of a specifically linked chain could be monitored. To test this, the NZF1 domain was expressed with an N-terminal fusion to HaloTag and 
covalently tethered to HaloLink resin as previously reported. ${ }^{38,40}$ The immobilized NZF1 domain was then used to isolate Ub chains from HEK293. As with TUBE2 enrichment, Western blot analysis shows the NZF1 domain enables isolation of high molecular weight chains (Figures 3, S1, and S2). Moreover, all three $\mathrm{Ub}_{1-74}$ species are detected by MS. Intriguingly, the Ub chain profile differs from that of the TUBE2 pull-downs. In unstimulated cells, approximately $4 \%$ of the NZF1-enriched chains contain branch points $\left({ }^{2 x G G} \mathrm{Ub}_{1-74}\right)$ compared to only $1 \%$ in TUBE2 pull-downs (Figure $3 \mathrm{~B}$ ). These differences are indeed significant based on the consistency of results obtained using different mass spectrometers (see below) and the number of biological and technical replicates. Narrowing the scope of chains that can be pulled down thus enables detection of chain architectures that might normally be lost in the noise.

Next, we sought to determine if NZF1-enriched chains are involved in proteasomal degradation. Again, HEK293 cells were treated with the proteasome inhibitor MG132 for 2, 4, 8, and $16 \mathrm{~h}$ (Figures 3 and S4 and Table S3). ${ }^{20}$ Interestingly, even after $16 \mathrm{~h}$, no significant changes in any of the three $\mathrm{Ub}_{1-74}$ species are detected. These results suggest chains enriched by the NZF1 domain do not target substrates to the proteasome.

\section{MS $^{2}$ Analysis of Branched Chains}

We sought to characterize the linkages in chains isolated from TUBE2 and NZF1 pulldowns. To this end, we employed electron-transfer dissociation (ETD) fragmentation to generate c- and $z^{\circ}$-type ions. ${ }^{41,42}$ ETD analysis of the ${ }^{\mathrm{GG}} \mathrm{Ub}_{1-74}$ species points to chains primarily composed of K48 linkages (Figure S5). Analysis of the ${ }^{2 \times G G} \mathrm{Ub}_{1-74}$ species, however, proved much more difficult, precluding us from identifying specific lysines that comprise the branch points (Figure S6). This could be due to the nonselective nature of TUBE2, which is likely to enrich for chains composed of multiple linkages.

As the NZF1 domain of TRABID has been shown to selectively isolate K29-linked Ub chains, we hoped to confirm the presence of K29 and identify other linkages within the branch points. Using the sample from MG132 treated cells, we isolated the $\mathrm{M}^{11+}$ charge state for ${ }^{2 \times G G} \mathrm{Ub}_{1-74}$ and subjected it to ETD fragmentation (Figure 4A). Accounting for all possible linkage types, the fragmentation pattern best fits a K29/K48 branch point. With these linkages, we identified $31 \mathrm{c}$ and $31 \mathrm{z}^{\bullet}$ ions (Figures 4B,C, S7, and S8). The most important ions for characterization are $\mathrm{c}_{31}$ and $\mathrm{z}_{27} 7^{\circ}$ (Figure $4 \mathrm{~B}$ ). The presence of these two indicates that the Gly-Gly modifications are on K29 and K48. These results are consistent with the previously reported observation that NZF1 enriches heterotypic chains comprised mainly of K29 and K48 linkages. ${ }^{38}$

To provide additional support for the presence of Gly-Gly modifications on K29 and K48, we used Ub chain restriction analysis (UbiCrest). ${ }^{43,44}$ NZF1-enriched chains were digested with the K29/K33 linkage-selective DUB TRABID and the K48 specific DUB OTUB1. ${ }^{45,46}$ As a control, we also employed the nonselective DUB USP15. ${ }^{47}$ Western blot analysis shows chains are sensitive to TRABID and OTUB1 with the greatest amount of cleavage occurring when both DUBs are added (Figure 4D). These data confirm that NZF1-enriched chains contain K29 and K48 linkages. Not all high-molecular weight species, however, are removed by TRABID and OTUB1. This suggests that proteins enriched by NZF1 are 
modified with multiple mono-Ub molecules or chains with linkages other than K29 and K48.

We then performed ETD analysis on the $\mathrm{M}^{11+}$ charge state of ${ }^{\mathrm{GG}} \mathrm{Ub}_{1-74}$ to identify other possible linkages. From the fragmentation patterns, it is evident that K48 linkages predominate (Figure S9), suggesting many of the isolated ubiquitylated proteins are modified with multiple mono Ub that are poor substrates for the linkage selective DUBs employed in our chain restriction digests. On the basis of these results along with the MS data, we propose that most of the isolated proteins are carrying multimono-Ub modifications along with short chains linked through K29 and K48. Our middle-down MS approach therefore informs on the overall landscape of Ub modifications and chain configuration.

\section{Ensuring Maximal Detection of the Ub Chain Population by UbiChEM-MS}

Optimizing the conditions for restricted trypsinolysis is critical for detecting all three $\mathrm{Ub}_{1-74}$ species. For instance, the use of denaturants commonly used to elute Ub chains from UBD resins must be avoided to ensure minimal cleavage. ${ }^{48}$ In our case, this was achieved by performing trypsinolysis reactions on resin. Another important issue is the amount of lysate required to observe low abundant modifications such as branching. We empirically found a ratio of 3-4 $\mu \mathrm{L}$ of resin per mg of lysate consistently leads to the detection of branched chains. Overdigestion of resin-bound chains is another problem we encountered. To avoid this, we performed a time course analysis to find the optimal trypsin concentration. As evidenced by the formation of di-Ub and mono-Ub, we observe complete conversion of high molecular weight chains within $16 \mathrm{~h}$ using $1 \mu \mathrm{g}$ of trypsin per $18 \mathrm{mg}$ of lysate for untreated cells and $1 \mu \mathrm{g}$ of trypsin per $6 \mathrm{mg}$ of lysate for cells treated with MG132 (Figures S1 and S10 and Table S1).

Pull-down efficiency is also a concern. This was assessed through recapture experiments in which fractions from a flow through were further incubated with a fresh batch of resin. We performed these experiments with cell lysates from both the 0 and $16 \mathrm{~h} \mathrm{MG132}$ treatments. Western blot analysis shows that for the untreated cells $(0 \mathrm{~h})$ most of the available Ub chains are captured (Figure S11). However, with cell lysates from the $16 \mathrm{~h}$ MG132 treatment, an appreciable amount of $\mathrm{Ub}$ species escape the first pull-down and are recaptured by fresh resin (Figure S11). This is not surprising considering MG132 treatment leads to a dramatic boost in the global levels of Ub chains, which will affect the Ub to resin ratio. ${ }^{12}$ Despite the inability to capture all the Ub chains in the initial round of pull-downs, middle-down MS shows the chains isolated from the second round have the same architecture as those from the first. These results indicate that our pull-down conditions are sufficient to yield a complete picture of the Ub landscape.

\section{UbiChEM-MS Is Not Limited by Instrumental Setup}

For the UbiChEM-MS workflow, we used different separation and characterization methods. With the TUBEs, online RPLC separation was coupled to a TOF mass spectrometer. With the NZF1 domain, RPLC was performed offline and analytes were then directly introduced into an Orbitrap mass spectrometer. Despite these differences, similar Ub architectures are 
observed from experiment-to-experiment and instrument-to-instrument. These results highlight the reproducibility and accessibility of our method.

\section{CONCLUSION}

Although a number of studies have implicated heterotypic Ub chains in the regulation of fundamental cellular pathways, the precise configuration of these chains has largely remained unknown. ${ }^{1-3,5,13}$ In this study, we established Ubiquitin Chain Enrichment Middle-down Mass Spectrometry (UbiChEM-MS), as a method for detecting and quantifying the relative abundance of heterotypic branched chains in cell lysates. Using two different UBDs, we demonstrate the dynamics of Ub branched chains containing K29 linkages as well as the global $\mathrm{Ub}$ pool in response to MG132 treatment. From these experiments, we found that, while chain branching does indeed increase in response to proteasome inhibition, branched chains containing K29 linkages are likely to be a nondegradative signal. Our work thus potentially expands the known role of branched Ub chains beyond just proteasome targeting signals.

Our strategy of using UBDs coupled with middle-down MS to detect and quantify relative amounts of branched chains has several advantages. First, it enables unambiguous detection of branching using endogenous levels of Ub chains. This avoids the use of Ub mutants, which may provide misleading results. Second, it exploits the selectivity of certain UBDs to examine the architecture of chains harboring specific linkages. Third, it distinguishes between mono-Ub/chain end-caps, the linear portion of a chain, and branch points. This is powerful because the relative changes in each of these species can be monitored under different cellular conditions.

\section{Supplementary Material}

Refer to Web version on PubMed Central for supplementary material.

\section{Acknowledgments}

This work was supported by research grant RO1GM110543 from the National Institutes of Health (NIH) and the National Science Foundation (NSF) Graduate Research Fellowship Program (GRFP, award number: 1451512). The purchase of the Bruker ULTRAFLEX III in 2008 was partially funded by NIH NCRR 1S10RR024601-01 Award to the University of Wisconsin-Madison Department of Chemistry. The data described herein were acquired on an Orbitrap Fusion mass spectrometer funded by National Institutes of Health grant 1S10OD010645-01A1.

\section{References}

1. Nakayama KI, Nakayama K. Nat Rev Cancer. 2006; 6:369-381. [PubMed: 16633365]

2. Jackson SP, Durocher D. Mol Cell. 2013; 49:795-807. [PubMed: 23416108]

3. Liu YC. Annu Rev Immunol. 2004; 22:81-127. [PubMed: 15032575]

4. Hershko A, Ciechanover A. Annu Rev Biochem. 1998; 67:425-479. [PubMed: 9759494]

5. Komander D, Rape M. Annu Rev Biochem. 2012; 81:203-229. [PubMed: 22524316]

6. Yau R, Rape M. Nat Cell Biol. 2016; 18:579-586. [PubMed: 27230526]

7. Swatek KN, Komander D. Cell Res. 2016; 26:399-422. [PubMed: 27012465]

8. Jin L, Williamson A, Banerjee S, Philipp I, Rape M. Cell. 2008; 133:653-665. [PubMed: 18485873] 
9. Chau V, Tobias J, Bachmair A, Marriott D, Ecker D, Gonda D, Varshavsky A. Science. 1989; 243:1576-1583. [PubMed: 2538923]

10. Thrower JS, Hoffman L, Rechsteiner M, Pickart CM. EMBO J. 2000; 19:94-102. [PubMed: 10619848]

11. Jacobson AD, Zhang N-Y, Xu P, Han K-J, Noone S, Peng J, Liu C-W. J Biol Chem. 2009; 284:35485-35494. [PubMed: 19858201]

12. Xu P, Duong DM, Seyfried NT, Cheng D, Xie Y, Robert J, Rush J, Hochstrasser M, Finley D, Peng J. Cell. 2009; 137:133-145. [PubMed: 19345192]

13. Haglund K, Dikic I. EMBO J. 2005; 24:3353-3359. [PubMed: 16148945]

14. Gerlach B, Cordier SM, Schmukle AC, Emmerich CH, Rieser E, Haas TL, Webb AI, Rickard JA, Anderton H, Wong WWL, Nachbur U, Gangoda L, Warnken U, Purcell AW, Silke J, Walczak H. Nature. 2011; 471:591-596. [PubMed: 21455173]

15. Ikeda F, Deribe YL, Skanland SS, Stieglitz B, Grabbe C, Franz-Wachtel M, van Wijk SJL, Goswami P, Nagy V, Terzic J, Tokunaga F, Androulidaki A, Nakagawa T, Pasparakis M, Iwai K, Sundberg JP, Schaefer L, Rittinger K, Macek B, Dikic I. Nature. 2011; 471:637-641. [PubMed: 21455181]

16. Kim HT, Kim KP, Lledias F, Kisselev AF, Scaglione KM, Skowyra D, Gygi SP, Goldberg AL. J Biol Chem. 2007; 282:17375-17386. [PubMed: 17426036]

17. Peng J, Schwartz D, Elias JE, Thoreen CC, Cheng D, Marsischky G, Roelofs J, Finley D, Gygi SP. Nat Biotechnol. 2003; 21:921-926. [PubMed: 12872131]

18. Kim HT, Kim KP, Uchiki T, Gygi SP, Goldberg AL. EMBO J. 2009; 28:1867-1877. [PubMed: $19387488]$

19. Grice GL, Lobb IT, Weekes MP, Gygi SP, Antrobus R, Nathan JA. Cell Rep. 2015; 12:545-553. [PubMed: 26190103]

20. Meyer HJ, Rape M. Cell. 2014; 157:910-921. [PubMed: 24813613]

21. Emmerich CH, Ordureau A, Strickson S, Arthur JSC, Pedrioli PGA, Komander D, Cohen P. Proc Natl Acad Sci U S A. 2013; 110:15247-15252. [PubMed: 23986494]

22. Emmerich CH, Bakshi S, Kelsall IR, Ortiz-Guerrero J, Shpiro N, Cohen P. Biochem Biophys Res Commun. 2016; 474:452-461. [PubMed: 27133719]

23. Boname JM, Thomas M, Stagg HR, Xu P, Peng J, Lehner PJ. Traffic. 2010; 11:210-220. [PubMed: 19948006]

24. Goto E, Yamanaka Y, Ishikawa A, Aoki-Kawasumi M, Mito-Yoshida M, Ohmura-Hoshino M, Matsuki Y, Kajikawa M, Hirano H, Ishido S. J Biol Chem. 2010; 285:35311-35319. [PubMed: 20833710]

25. Saeki Y, Tayama Y, Toh-e A, Yokosawa H. Biochem Biophys Res Commun. 2004; 320:840-845. [PubMed: 15240124]

26. Koegl M, Hoppe T, Schlenker S, Ulrich HD, Mayer TU, Jentsch S. Cell. 1999; 96:635-644. [PubMed: 10089879]

27. Xu P, Peng J. Anal Chem. 2008; 80:3438-3444. [PubMed: 18351785]

28. Valkevich EM, Sanchez NA, Ge Y, Strieter ER. Biochemistry. 2014; 53:4979-4989. [PubMed: 25023374]

29. Lee AE, Castañeda CA, Wang Y, Fushman D, Fenselau C. J Mass Spectrom. 2014; 49:1272-1278. [PubMed: 25476945]

30. Lee AE, Geis-Asteggiante L, Dixon EK, Kim Y, Kashyap TR, Wang Y, Fushman D, Fenselau C. J Mass Spectrom. 2016; 51:315-321. [PubMed: 27041663]

31. Lee AE, Geis-Asteggiante L, Dixon EK, Miller M, Wang Y, Fushman D, Fenselau C. J Mass Spectrom. 2016; 51:629-637. [PubMed: 28239975]

32. Wilkinson KD, Audhya TK. J Biol Chem. 1981; 256:9235-9241. [PubMed: 6267067]

33. Husnjak K, Dikic I. Annu Rev Biochem. 2012; 81:291-322. [PubMed: 22482907]

34. Guner H, Close PL, Cai W, Zhang H, Peng Y, Gregorich ZR, Ge Y. J Am Soc Mass Spectrom. 2014; 25:464-470. [PubMed: 24385400]

35. Peng Y, Gregorich ZR, Valeja SG, Zhang H, Cai W, Chen Y-C, Guner H, Chen AJ, Schwahn DJ, Hacker TA, Liu X, Ge Y. Mol Cell Proteomics. 2014; 13:2752-2764. [PubMed: 24969035] 
36. Chen YC, Ayaz-Guner S, Peng Y, Lane NM, Locher MR, Kohmoto T, Larsson L, Moss RL, Ge Y. Anal Chem. 2015; 87:8399-8406. [PubMed: 26189812]

37. Hjerpe R, Aillet F, Lopitz-Otsoa F, Lang V, England P, Rodriguez MS. EMBO Rep. 2009; 10:1250-1258. [PubMed: 19798103]

38. Kristariyanto YA, Rehman SAA, Campbell DG, Morrice NA, Johnson C, Toth R, Kulathu Y. Mol Cell. 2015; 58:83-94. [PubMed: 25752573]

39. Scott D, Oldham NJ, Strachan J, Searle MS, Layfield R. Proteomics. 2015; 15:844-861. [PubMed: 25327553]

40. Los GV, Encell LP, McDougall MG, Hartzell DD, Karassina N, Zimprich C, Wood MG, Learish R, Ohana RF, Urh M, Simpson D, Mendez J, Zimmerman K, Otto P, Vidugiris G, Zhu J, Darzins A, Klaubert DH, Bulleit RF, Wood KV. ACS Chem Biol. 2008; 3:373-382. [PubMed: 18533659]

41. Zubarev RA, Kelleher NL, McLafferty FW. J Am Chem Soc. 1998; 120:3265-3266.

42. Zubarev RA, Horn DM, Fridriksson EK, Kelleher NL, Kruger NA, Lewis MA, Carpenter BK, McLafferty FW. Anal Chem. 2000; 72:563-573. [PubMed: 10695143]

43. Mevissen TET, Hospenthal MK, Geurink PP, Elliott PR, Akutsu M, Arnaudo N, Ekkebus R, Kulathu Y, Wauer T, El Oualid F, Freund SMV, Ovaa H, Komander D. Cell. 2013; 154:169-184. [PubMed: 23827681]

44. Hospenthal MK, Mevissen TET, Komander D. Nat Protoc. 2015; 10:349-361. [PubMed: 25633630]

45. Virdee S, Ye Y, Nguyen DP, Komander D, Chin JW. Nat Chem Biol. 2010; 6:750-757. [PubMed: 20802491]

46. Wang T, Yin L, Cooper EM, Lai MY, Dickey S, Pickart CM, Fushman D, Wilkinson KD, Cohen RE, Wolberger C. J Mol Biol. 2009; 386:1011-1023. [PubMed: 19211026]

47. Ritorto MS, Ewan R, Perez-Oliva AB, Knebel A, Buhrlage SJ, Wightman M, Kelly SM, Wood NT, Virdee S, Gray NS, Morrice NA, Alessi DR, Trost M. Nat Commun. 2014; 5:4763. [PubMed: 25159004]

48. Emmerich CH, Cohen P. Biochem Biophys Res Commun. 2015; 466:1-14. [PubMed: 26325464] 


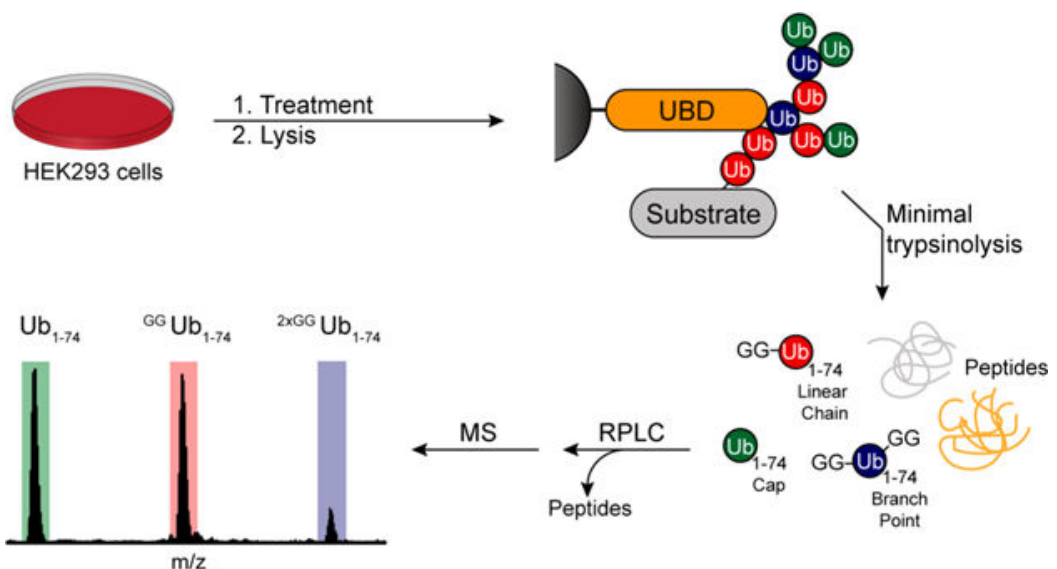

Figure 1.

General workflow for isolation and detection of branched Ub chains by UbiChEM-MS. Cells are grown under different treatments and lysed under nondenaturing conditions. Clarified lysates are subsequently incubated with a resin bearing ubiquitin binding domains, and the isolated chains are then subjected to minimal trypsinolysis under nondenaturing conditions yielding $\mathrm{Ub}_{1-74}$ fragments containing Gly-Gly modifications. These products are enriched for using reverse-phase chromatography and characterized by high resolution mass spectrometry. 


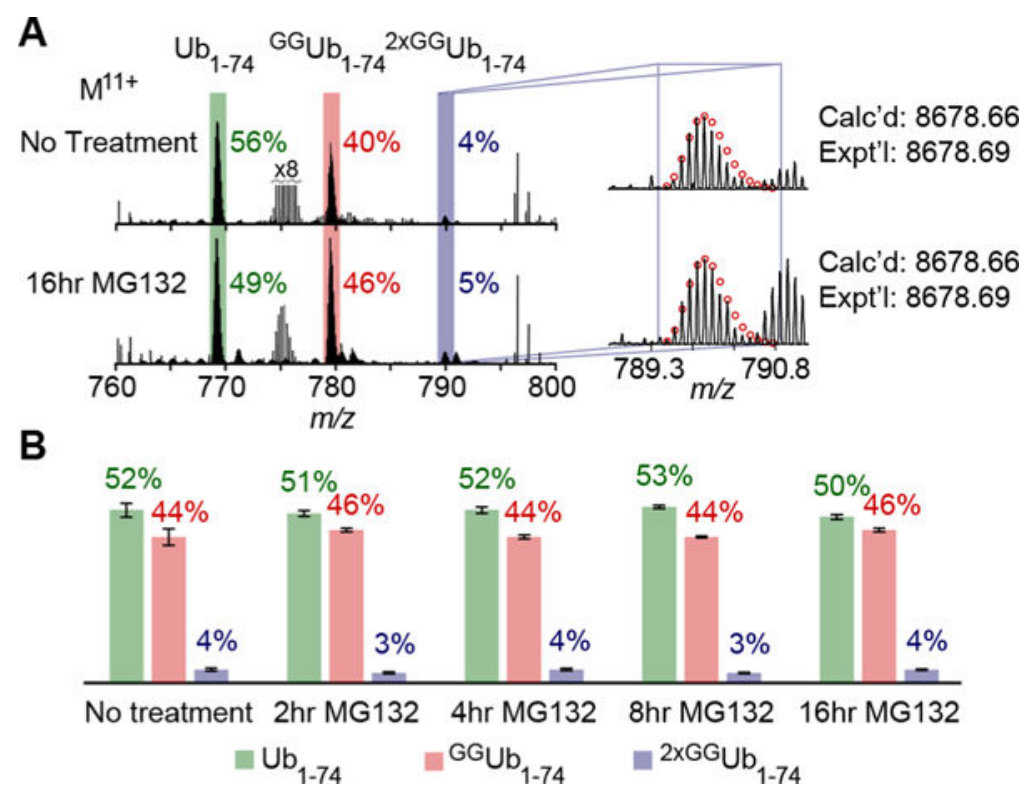

Figure 2.

Middle-down MS analysis of Ub chains isolated from HEK cells using agarose bound TUBE2 shows dynamics in Ub chain branching upon proteasome inhibition. (A) ESI TOF MS spectra showing the presence of all three minimally digested products: $\mathrm{Ub}_{1-74}$ (green

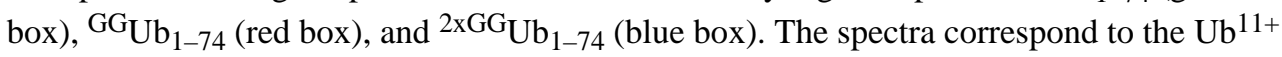
charge state for chains isolated from either untreated or MG132 treated HEK lysate. (B) Quantification of $\mathrm{Ub}_{1-74}$ species at different MG132 time points. The percent distribution is calculated by averaging relative abundance of each $\mathrm{Ub}_{1-74}$ species to total abundance of all three species from three biological replicates for each treatment (Table S2). Error bars represent standard error of the mean (SEM) for each data set: *, $p<0.05$; **, $p<0.01$; ***, $p<0.001$ (Student's $t$ test). Calcd, calculated most abundant weight; exptl, experimental most abundant molecular weight. 

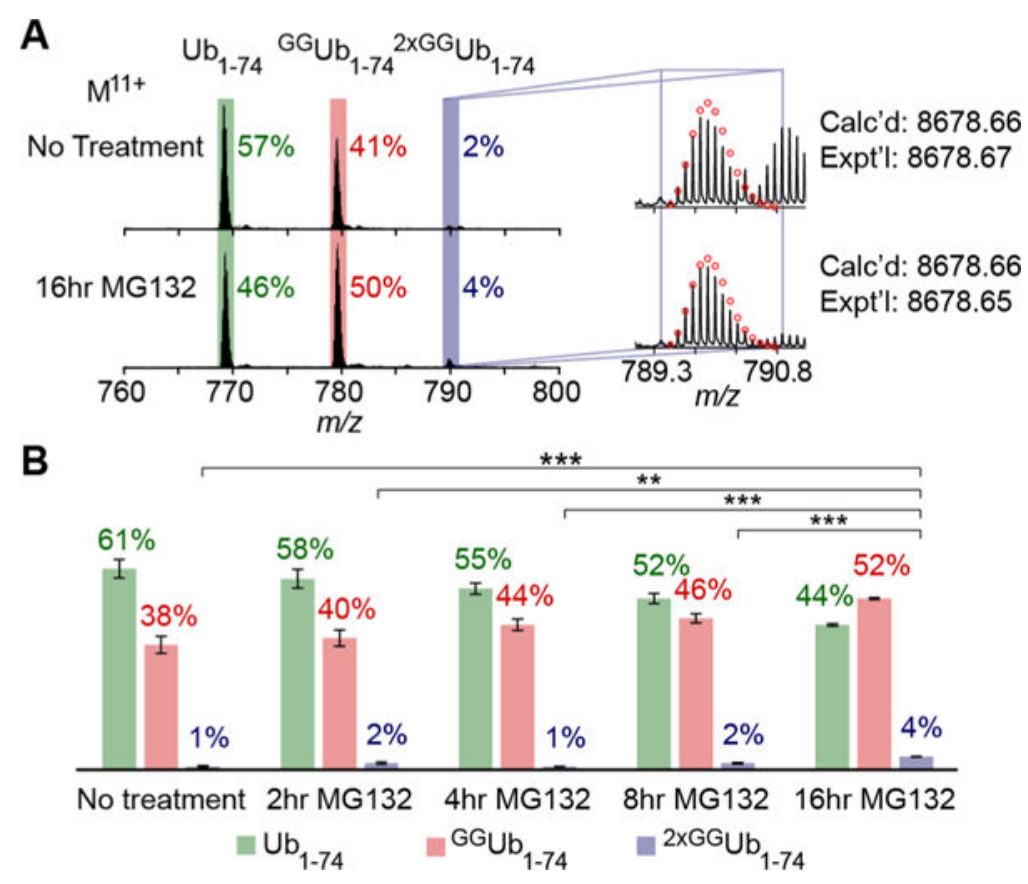

Figure 3.

Middle-down MS analysis of Ub chains isolated from HEK cells using Halo-NZF1 resin. (A) ESI MS spectra showing the MS spectra of chains isolated using Halo-NZF1 resin. The spectra shows the presence of all three minimally digested products: $\mathrm{Ub}_{1-74}$ (green

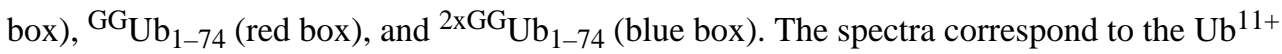
charge state for chains isolated from either untreated or MG132 treated HEK lysate. (B) Quantification of $\mathrm{Ub}_{1-74}$ species after different lengths of proteasome inhibition. The percent distribution is calculated by averaging relative abundance of each $\mathrm{Ub}_{1-74}$ species to total abundance of all three species from three biological replicates for each treatment (Table S3). Error bars represent the SEM for each data set. Calcd, calculated most abundant weight; exptl, experimental most abundant molecular weight. 

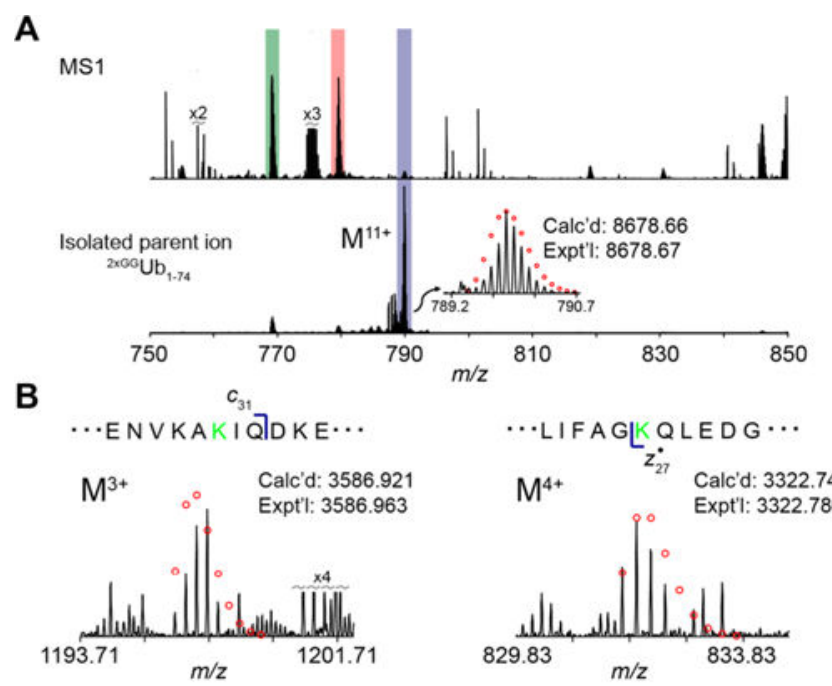

C
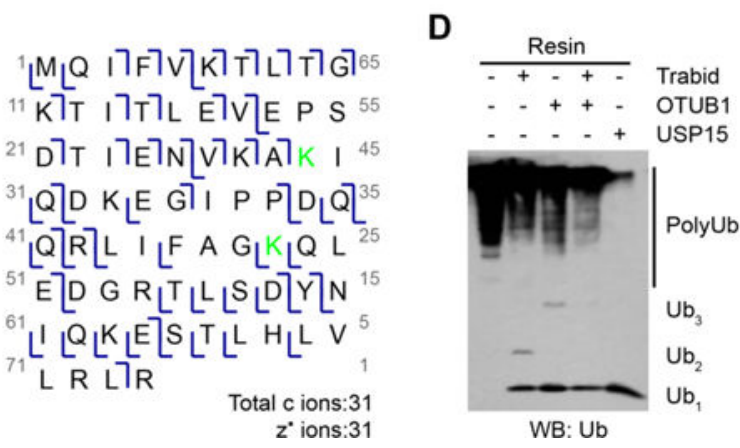

Figure 4.

Characterization of Ub chains isolated with Halo-NZF1 by ETD and DUB restriction analysis uncovering K29/K48 branched chains. (A) ESI MS analysis of the minimally digested Ub chains isolated from HEK cells treated with $10 \mu \mathrm{M}$ MG132 for $2 \mathrm{~h}$. The spectra correspond to the $\mathrm{Ub}^{11+}$ charge state, where the top spectrum shows all ions present in the mass range and the bottom spectrum shows the isolated ${ }^{2 x_{G G}} U_{1-74} M^{11+}$ parent ion. The red circles represent theoretical isotopic abundance distributions of the isotopomer peaks. calcd, calculated most abundant weight; exptl, experimental most abundant molecular weight. (B) Analysis of ETD fragments showing the presence of a di-Gly modification at K29 and K48. The green K shows the position of a modified lysine. (C) Observed ETD fragments ( $c$ and $\mathrm{z}^{\bullet}$ ions) mapped onto the sequence of $\mathrm{Ub}$ containing a di-Gly modification at K29 and K48. (D) DUB restriction analysis of Ub chains isolated from MG132 treated cells using Halo-NZF1 resin. Aliquots of this resin were incubated with the indicated DUBs overnight at $37^{\circ} \mathrm{C}$. The reaction mixtures were quenched with $6 \times$ Laemmli loading dye, separated on a $15 \%$ SDS-PAGE gel, and analyzed by Western blot using anti-Ub antibody (P4D1). 